\title{
ENHANCING SELECTIVITY OF CANTILEVER-BASED RESONANT CHEMICAL SENSORS THROUGH TRANSIENT MEASUREMENTS AT ELEVATED TEMPERATURES
}

\author{
P. Getz ${ }^{l}$, C. Carron $^{l, 2}$, and O. Brand ${ }^{l}$ \\ ${ }^{1}$ School of Electrical and Computer Engineering, Georgia Institute of Technology, Atlanta, GA, USA \\ ${ }^{1}$ Institute for Electronics and Nanotechnology, Georgia Institute of Technology, Atlanta, GA, USA \\ ${ }^{2}$ Harris Corporation, Melbourne, FL, USA
}

\begin{abstract}
This paper introduces a method to increase the selectivity of mass-sensitive chemical sensors towards volatile organic compounds (VOCs) by analyzing transient data as a function of device temperature. Heating pulses applied to a resonant, masssensitive cantilever cause a $3{ }^{\circ} \mathrm{C}$ to $30{ }^{\circ} \mathrm{C}$ rise in the sensing film temperature and, thus, a reduction in the partition coefficient, resulting in analyte desorption at the elevated temperature. Extracted temperature-dependent diffusion rates can be fit to an Arrhenius-type relationship whose coefficients can be used for analyte discrimination.
\end{abstract}

\section{INTRODUCTION}

In recent years, microelectromechanical systems (MEMS) based sensing devices have been incorporated into a variety of products, often as an inexpensive alternative to benchtop devices. While MEMS-based chemical sensors typically cannot offer superior performance (measured by selectivity, sensitivity and stability) compared to traditional desktop or laboratory units, MEMS solutions can be designed for applications where system requirements exclude traditional instrumentation, such as gas chromatography and mass spectroscopy units, due to their high cost, limited portability and high-power requirements $[1,2]$.

Many MEMS-based chemical sensors rely on analyte sorption into a polymeric sensing film [3]. Because these polymer sensing films are only partially selective to any analyte, sensors relying on analyte absorption into the polymer sensing film as a single data point face inherent uncertainty [4,5]. As such, often arrays of sensors coated with different partially-selective films are used to decrease the uncertainty and improve the selectivity of the sensing system [6-8].

Various methods have been previously explored to increase the selectivity of a chemisorbing sensor via analyzing acquired transient data. Kummer et al. and Su et al. demonstrated the effectiveness of using a valve-generated signal transient to discriminate between similar analytes in a chemicapacitor [9] and resonant cantilever [3], respectively. Traditional valves that generate analyte absorption transients can be replaced with integrated heating resistors, which enable analyte desorption from the sensing film via the temperaturedependence of the partition coefficient [10]. Moreover, it is well known that diffusion rates of gaseous chemicals into a sensing film exhibit an Arrhenius relationship with temperature [9,11]. Building on these efforts, this work explores analyte sorption transients recorded at different elevated temperatures to extract the analytespecific (for a particular sensing film) coefficients of the Arrhenius relationship and improve the sensor selectivity.

\section{THEORY}

Analyte absorption and desorption into a sensing film generally follows Fick's law of diffusion and can be simplified, for most chemical sensors, by considering one-dimensional diffusion into a film terminated at one end. The normalized mass gained by this film is given by [3]

$$
\frac{M(t)}{M_{\infty}}=1-8 \cdot \sum_{n=0}^{\infty} \frac{1}{[(2 n+1) \pi]^{2}} e^{-(t / 4 \tau)[(2 n+1) \pi]^{2}}
$$

where $M(t)$ is the mass gained or lost at time, $t$, and $M_{\infty}$ is the equilibrium mass gained or lost. The characteristic diffusion time, $\tau$, is defined as

$$
\tau=h^{2} / D(T)
$$

where $h$ is the film thickness and the diffusion coefficient, $D$, is a function of temperature in Kelvin, $T$. For the initial mass gain or loss, this infinite sum can be approximated as

$$
\frac{M(t)}{M_{\infty}}=\frac{2}{h} \sqrt{\frac{D(T) \cdot t}{\pi}}
$$

for $M(t) / M_{\infty} \leq 0.5$. Thus, the temperature-dependent diffusivity coefficients can be estimated from measured sensor transients.

The temperature dependence of the analyte diffusivity into a polymer sensing film can be approximated by the linearized form of the Arrhenius relationship [9]

$$
\ln D(T)=-\frac{E}{R}\left(\frac{1}{T}\right)+\ln D_{0}
$$

where the measured diffusivity from Eq. (3) is a function of the film temperature, the activation energy for diffusion of analyte molecules into the sensing film, $E$, the gas constant, $R$, and the Arrhenius preexponential factor, $D_{0}$, relating to the diffusivity as temperature approaches infinity.

\section{SENSING SYSTEM}

Figure 1 shows the heated mass-sensitive chemical sensor used for this work, comprising a semi-circular hammerhead-like suspended resonator fabricated in the device layer of an SOI wafer. The fabrication procedure follows the process developed for

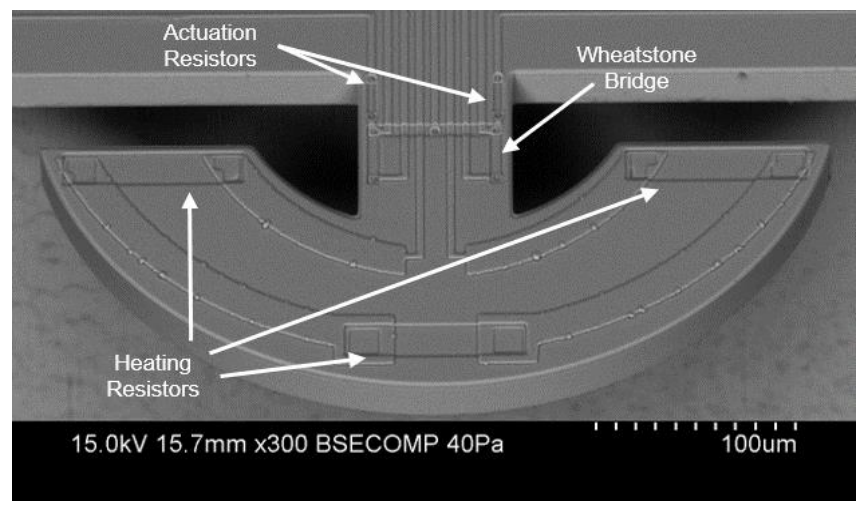

Figure 1: SEM micrograph of a resonant cantilever with a nearsemicircular head supported by a $75 \mu \mathrm{m}$ wide, $100 \mu \mathrm{m}$ long beam. Resistors for thermal excitation and piezoresistive detection of inplane vibrations are located at the cantilever base. Three heating resistors, for thermal modulation, are visible on the head region. 


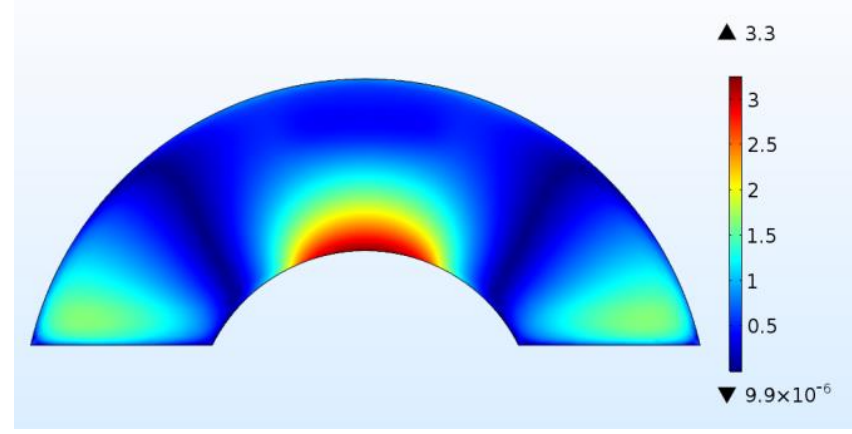

Figure 2: COMSOL finite element model analysis of temperature uniformity within a $5 \mu \mathrm{m}$ PIB sensing film: displayed is the absolute deviation in percent from the mean film temperature due to thermal modulation from the integrated heating resistors on the heated cantilever's hammerhead region.

resonant chemical sensors in our group $[10,12]$. The hammerhead region is spray-coated via shadow masking with an appropriate sensing film, polyisobutylene (PIB) in the present work, for the analytes of interest. The hammerhead region is anchored via a 100 $\mu \mathrm{m}$ long, $75 \mu \mathrm{m}$ wide beam and the sensor's resonance frequency prior to coating is $740-760 \mathrm{kHz}$. Three diffused resistors in the hammerhead region enable rapid, localized heating to modulate the sorption kinetics and generate temperature-modulated sorption transients.

The device's geometry seeks to maintain thermal uniformity within the coated hammerhead region, maximize heating power efficiency while minimizing thermal rise times. Additionally, the heating resistors' location at the distal end of the cantilever minimizes temperature rise near the beam's anchor point and limits the effects of self-heating on the resonant frequency shift due to the temperature dependency of silicon's Young's modulus.

A COMSOL finite element analysis of the steady-state temperature elevation of the device with coated film in Figure 2 shows the deviation in percent from the average film temperature. The analysis highlights a fairly uniform film temperature with a $<1 \%$ deviation from the mean temperature rise for $66 \%$ of the film and a $<3 \%$ deviation for $99.8 \%$ of the film. This ensures a consistent partition coefficient and diffusivity coefficient throughout the absorbing film.

Figure 3 shows the normalized simulated thermal transient of the heated hammerhead structure and coated sensing film from a COMSOL simulation for a $6 \mu \mathrm{m}$ PIB coated device. The $90 \%$ thermal rise time of the coated film is approximately $3 \mathrm{~ms}$. Since typical absorption transients for this polymer thickness are expected

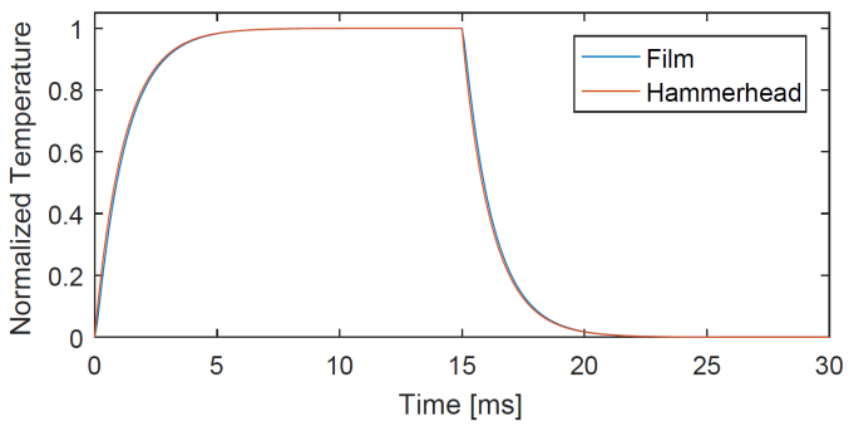

Figure 3: COMSOL finite element simulation of the normalized temperature transient of the silicon hammerhead region (red line) and the $5 \mu \mathrm{m}$ PIB sensing film (blue line) for a $15 \mathrm{~ms}$ heating pulse applied to the heating resistors. to be on the order of tens to hundreds of seconds, such short thermal transients of the device ensure that they will not influence the measured sorption transients or extracted Arrhenius coefficients.

\section{EXPERIMENTAL}

The gas mixing setup used has been described in detail by $\mathrm{Su}$ [3]. It features multiple mass flow controllers and glass bubblers with liquid analyte for a carrier gas (nitrogen) to flow through to control analyte and reference gas streams. A high-speed pneumatic four-way valve switches between an ultra-high purity nitrogen reference gas stream and an analyte-loaded gas stream from the liquid bubbler (set in a temperature-controlled bath) that has been diluted from its Antoine equation partial pressure by nitrogen. The setup enables analyte concentrations from hundreds to thousands of ppm, depending on the analyte's partial pressure.

The hammerhead resonators are embedded in an amplifying feedback loop on a dedicated printed circuit board that ensures continuous operation at their fundamental in-plane resonance frequency. The resonance frequencies of a coated and an uncoated cantilever are measured simultaneously with a frequency counter. The uncoated cantilever compensates for environmental fluctuations within the sensing chamber not relating to analyte sorption. The LabVIEW-controlled setup measures the devices' resonant frequencies at 2-5 samples per second.

On experiment start, the system flows pure carrier gas over the resonator until equilibrium is reached. A chemical measurement begins as the four-way valve switches from carrier gas to analyte loaded gas. After the room-temperature analyte-loaded equilibrium is reached, a voltage is applied to the integrated resistors for a set time via a function generator or source-meter, thus modulating the sensing film's temperature with the applied power. The elevated temperature reduces the film's partition coefficient, causing analyte to desorb out of the sensing film. After a set heating period (10 minutes for the experiments in this work), the heating voltage is turned off and the film returns to its original temperature and partition coefficient. This causes the analyte to re-absorb into the sensing film. This sequence is repeated multiple times with various heating powers $(5 \mathrm{~mW}, 10 \mathrm{~mW}, 20 \mathrm{~mW}$, and $40 \mathrm{~mW})$ to generate data points for the Arrhenius relationship fit.

Upon conclusion of the heating pulse chain, the valve system changes back to the pure carrier gas flow and the system is brought back to equilibrium as a reset prior to subsequent measurements. Figure 4 shows the response of the sensor to heating pulses under

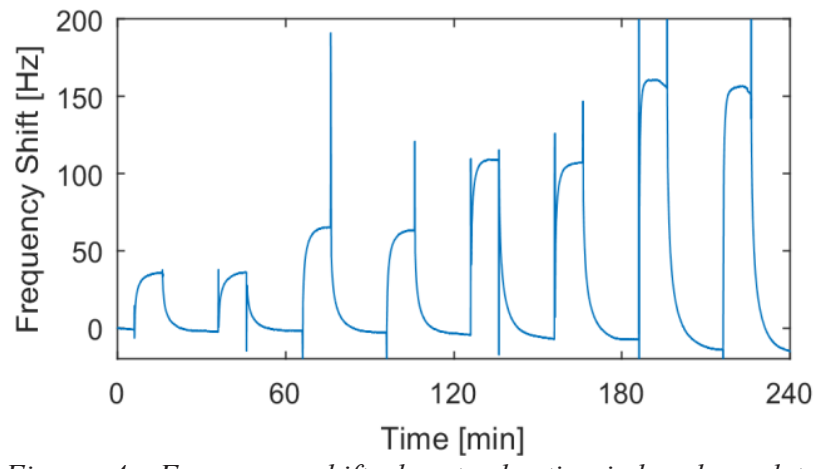

Figure 4: Frequency shift due to heating-induced analyte desorption for two 10-minute long heating pulses each at a heating power of 5, 10, 20 and $40 \mathrm{~mW}$. The resonator is coated with a PIB sensing film and exposed to a constant $630 \mathrm{ppm}$ concentration of ethyl-benzene during the 4-hour measurement period. Signal spikes at the beginning/end of the heating pulses are artifacts stemming from signal analysis and limited sampling rate. 


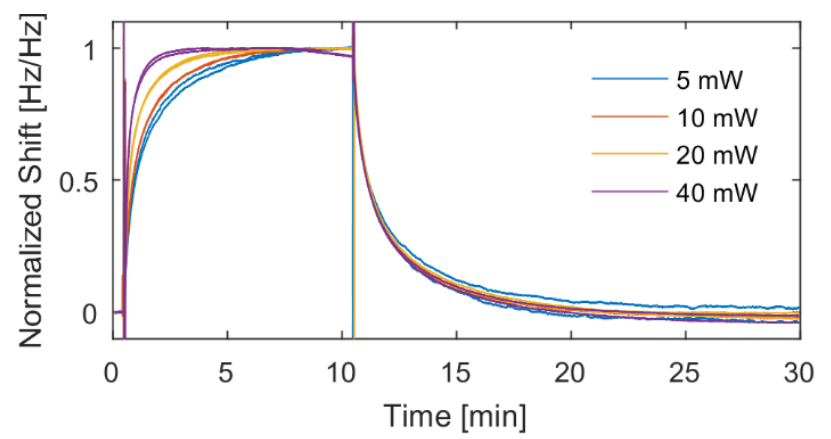

Figure 5: Measured normalized sensor frequency shift due to analyte sorption during 10-minute heating pulses (5, 10, 20 and 40 $\mathrm{mW}$ pairs). A positive shift indicates desorption due to the increased temperature, while a negative shift after a heating pulse indicates re-absorption into the sensing film. Desorption transients show different time constants at different temperatures because of the temperature dependence of the analyte diffusivity in the sensing film. The re-absorption is happening at room temperature and thus exhibits the same time constant for all heating pulses.

approximately $630 \mathrm{ppm}$ ethyl-benzene exposure after the effect of device heating on the sensor response is removed in post-processing. This procedure is repeated for the five other analytes in this work: pentane, chloroform, benzene, toluene and o-xylene. The tests with different analyte were performed sequentially using the same 6.1 $\mu \mathrm{m}$ thick PIB sensing film.

Figure 5 overlays the responses of the sensor with a $6.1 \mu \mathrm{m}$ thick PIB sensing film for different heating pulse powers. The data is normalized based on the steady-state frequency shift for each heating power (which can also be used as an independent metric to increase selectivity). The differing diffusion time coefficients are easily distinguishable during the initial 10 -minute heating phase and can be fit to the Arrhenius curve via a first order polynomial fit $(y=m x+b)$ according to (4).

\section{RESULTS}

Figures 6 and 7 show the extracted diffusivity data as a function of temperature and the logarithm of the diffusivity as a function of the inverse temperature (according to Eq. (4)), respectively. Table I summarizes the extracted numerical values for $E$ and $D_{0}$ for the tested six volatile organic compounds. Thereby, the temperature

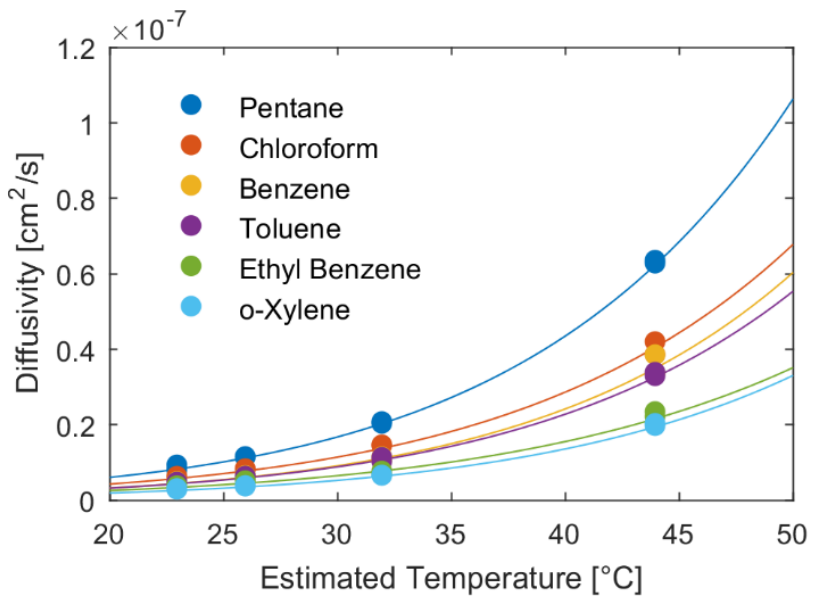

Figure 6: Extracted diffusivity for six different analytes in a PIB sensing film as a function of temperature. The measured data points are fitted to the Arrhenius equation (solid lines).

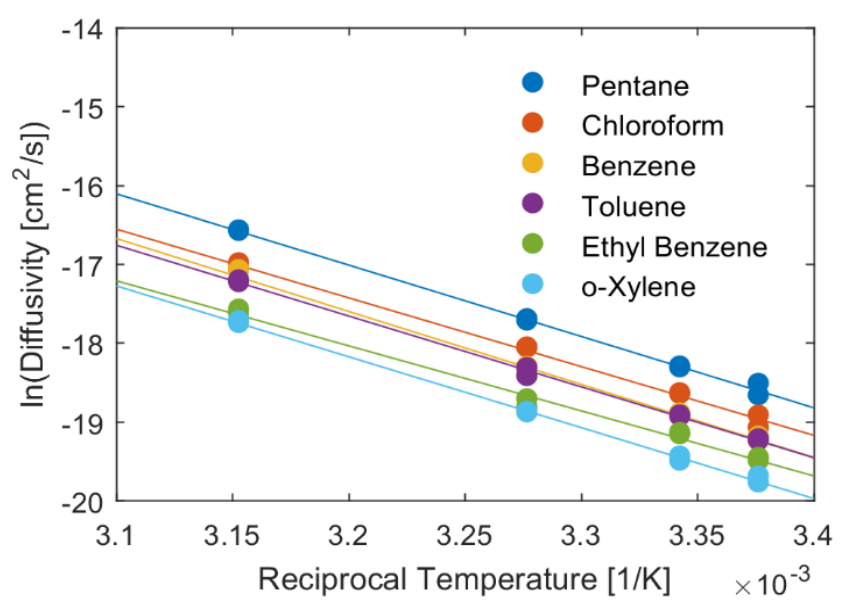

Figure 7: Logarithm of extracted diffusivity as a function of the inverse sensing film temperature for six different analytes in a PIB sensing film. According to the linearized form of the Arrhenius equation (see Eq. (4)), different $\mathrm{D}_{0}$ are seen as different $y$ intercepts, while different activation energies $\mathrm{E}$ result in different slopes.

rise per applied heating power is estimated from COMSOL simulations and calibration measurements in an environmental chamber to be $0.6{ }^{\circ} \mathrm{C} / \mathrm{mW}$. Clear numerical differentiation, particular in case of $D_{0}$, shows the ability of this method to distinguish between analytes. In case of similar $D_{0}$, the activation energy, $E$, assists to discriminate between analytes, enhancing the ability of sensors to distinguish between different analytes with similar diffusivity constants at room temperature.

The extracted data suggests that even chemically similar analytes, such as benzene, toluene, o-xylene and ethyl-benzene, can be distinguished using the presented method. These analytes have a common benzene ring and differ with the addition of carbon groups added to the ring. Optimization of polymer thickness and type can further target specific analytes and lead to an improved sensor performance with improved selectivity based solely on the temperature dependence of the analyte diffusivity.

Table I: Summary of extracted Arrhenius diffusivity coefficients $D_{0}$ and $E$ for six analytes in a PIB sensing film. Differentiation in both $D_{0}$ and $E$ lead to an increased ability to distinguish analytes from one another and increases sensor selectivity.

\begin{tabular}{|lrr|}
\hline Analyte & $\mathbf{D}_{\mathbf{0}}\left[\mathbf{m}^{\mathbf{2}} / \mathbf{s e c}\right]$ & $\mathbf{E}[\mathbf{k J} / \mathbf{m o l}]$ \\
\hline Benzene & 8.159 & 75.16 \\
Chloroform & 1.897 & 70.93 \\
Ethyl Benzene & 0.304 & 67.71 \\
Pentane & 16.91 & 75.47 \\
Toluene & 6.368 & 74.61 \\
o-Xylene & 2.801 & 73.85 \\
\hline
\end{tabular}

\section{CONCLUSION}

This paper demonstrates the design of a chemisorbing masssensitive heated cantilever and method to increase system selectivity via analyzing recorded analyte transients as a function of sorbing film temperature. Diffusivity coefficients extracted from analyte desorption transients, induced by a temperature increase of the sensing film, for different volatile organic compounds were fitted to an Arrhenius relationship. This relationship yielded parameters that can help distinguish between analytes and, thus, improve sensor selectivity. In future work, the sensor response to analyte mixture will be investigated. 


\section{ACKNOWLEDGEMENTS}

This work was performed at Georgia Tech's Institute for Electronics and Nanotechnology (IEN), a member of the National Nanotechnology Coordinated Infrastructure (NNCI), which is supported by the National Science Foundation (Grant ECCS1542174). The authors would like to thank the IEN staff for their assistance during device fabrication and testing.

\section{REFERENCES}

[1] C. K. Ho, A. Robinson, D. R. Miller, and M. J. Davis, "Overview of sensors and needs for environmental monitoring," Sensors, vol. 5, no. 1, pp. 4-37, 2005.

[2] C. K. Ho, M. T. Itamura, M. Kelley, and R. C. Hughes, "Review of chemical sensors for in-situ monitoring of volatile contaminants," Sandia Report, 2001.

[3] J.-J. Su, C. Carron, S. Truax, K. Demirci, L. Beardslee, and $\mathrm{O}$. Brand, "Assessing polymer sorption kinetics using micromachined resonators," in Solid-State Sensors, Actuators and Microsystems Conference (TRANSDUCERS), 2011 16th International, 2011, pp. 1420-1423: IEEE.

[4] A. Hierlemann, A. J. Ricco, K. Bodenhöfer, A. Dominik, and W. Göpel, "Conferring selectivity to chemical sensors via polymer side-chain selection: thermodynamics of vapor sorption by a set of polysiloxanes on thicknessshear mode resonators," Analytical chemistry, vol. 72, no. 16, pp. 3696-3708, 2000.

[5] A. Hierlemann, E. T. Zellers, and A. J. Ricco, "Use of linear solvation energy relationships for modeling responses from polymer-coated acoustic-wave vapor sensors," Analytical chemistry, vol. 73, no. 14, pp. 34583466, 2001.

[6] F. Battiston et al., "A chemical sensor based on a microfabricated cantilever array with simultaneous resonance-frequency and bending readout," Sensors and Actuators B: Chemical, vol. 77, no. 1-2, pp. 122-131, 2001.
[7] G. Yoshikawa et al., "Sub-ppm detection of vapors using piezoresistive microcantilever array sensors," Nanotechnology, vol. 20, no. 1, p. 015501, 2008.

[8] A. S. Pavluchenko, A. V. Mamykin, A. L. Kukla, N. V. Konoshchuk, O. Y. Posudievsky, and V. G. Koshechko, "Estimation of multicomponent organic solvent vapor mixture composition with electroconducting polymer chemiresistors," Sensors and Actuators B: Chemical, vol. 232, pp. 203-218, 2016.

[9] A. M. Kummer, T. P. Burg, and A. Hierlemann, "Transient signal analysis using complementary metal oxide semiconductor capacitive chemical microsensors," Analytical chemistry, vol. 78, no. 1, pp. 279-290, 2006.

[10] C. Carron, P. Getz, S. M. Heinrich, F. Josse, and O. Brand, "Cantilever-based resonant microsensors with integrated temperature modulation for transient chemical analysis," in Transducers 2015, 18th International Conference on Solid-State Sensors, Actuators and Microsystems, 2015, pp. 1511-1514.

[11] J. L. Duda, J. S. Vrentas, S. T. Ju, and H. T. Liu, "Prediction of diffusion coefficients for polymer-solvent systems," AIChE Journal, vol. 28, no. 2, pp. 279-285, 1982.

[12] L. A. Beardslee, A. M. Addous, S. Heinrich, F. Josse, I. Dufour, and O. Brand, "Thermal Excitation and Piezoresistive Detection of Cantilever In-Plane Resonance Modes for Sensing Applications," Microelectromechanical Systems, Journal of, vol. 19, no. 4, pp. 1015-1017, 2010.

\section{CONTACT}

P. Getz, tel: +1-916-850-5585; patrick.getz@gatech.edu

O. Brand, tel: +1-404-894-9425; oliver.brand@ece.gatech.edu 